“ (C) 2016 IEEE. Personal use of this material is permitted. Permission from IEEE must be obtained for all other uses, in any current or future media, including

reprinting/republishing this material for advertising or promotional purposes, creating new collective works, for resale or redistribution to servers or lists, or reuse of any copyrighted component of this work in other works." 


\title{
Controlling the shape and scale of triangular formations using landmarks and bearing-only sensing
}

\author{
Isaac L. Manuel, Adrian N. Bishop, Brian D.O. Anderson and Changbin Yu
}

\begin{abstract}
This work considers the scenario where three agents that can sense only bearings use two landmarks to control their formation shape. We define a method of relating the known distance separating the landmarks back to the edge lengths of the triangular formation. The result is used to define a formation control law that incorporates inter-agent distance constraints. We prove a strong exponential convergence result and show how one can extend the controller such that global stability from any initial position is possible.
\end{abstract}

\section{INTRODUCTION}

This work describes a method for controlling the formation shape $^{1}$ of three autonomous vehicles equipped only with passive sensors. The method requires that the autonomous vehicles (agents) are capable of measuring local bearings ${ }^{2}$ to landmarks and to other agents in the formation. By knowing the location of two landmarks it is possible to calculate the distance separating two agents and once the inter-agent distances are known then we can define a control law at each agent that forces that agent to achieve and then maintain desired inter-agent distance values. We can also extend this control law such that each agent maintains the interior angle subtended at itself by the other agents at some desired value (consistent with the desired distances). The end result is an exponentially stable formation control law with a global region of convergence.

Formation control is important for many real world applications of cooperative systems [1]; e.g. arranging mobile sensors for target localisation [2], [3]. The high-level goal of this research is to control formations in an efficient manner, as observed in nature, for a useful purpose. In 1987 Reynolds [4] simulated natural swarming (flocks, herds, and schools) using a distributed behavioural model. Other early work included that of Vicsek et al. [5] who modelled biological

I.L. Manuel was a summer research scholar with the Research School of Engineering at the Australian National University (ANU) and with NICTA.

A.N. Bishop is with the University of Technology Sydney (UTS) and NICTA, Canberra Research Lab. He is also an adjunct fellow at the ANU. E-mail: adrian.bishop@uts.edu.au

B.D.O. Anderson is with the ANU and NICTA. C. Yu is with the ANU and Shandong Computer Science Centre in Jinan, China.

This work was supported by NICTA and the Australian Research Council (ARC) via DE-120102873 and DP-110100538. A.N. Bishop is an ARC DECRA Fellow and C. Yu is an ARC Queen Elizabeth II Fellow. C. Yu is also supported by the Overseas Expert Program of Shandong Province.

${ }^{1}$ Throughout this work, formation shape will refer to both the shape and scale of the formation. Typically agents that sense and control only relative bearings cannot control the scale of the formation. This paper goes further by exploiting bearing measurements to landmarks with known positions in order to control both formation scale and shape.

${ }^{2}$ Bearing measurements at each agent are taken in a local coordinate frame; i.e. there is no reference to a common direction known by all agents. particles that exhibited cooperative behaviour. Both publications modelled the aggregate motion of a group of agents that arose as a result of local interactions between individual animals or particles respectively. In 2003, Jadbabaie et al. [6] offered a theoretical explanation of the behaviour exhibited by the simulation of Vicsek et al.

Modern formation control is often achieved through the use of the global positioning system (GPS). GPS has been used in formation control by [7] for controlling autonomous vehicles using GPS equipped surface craft, and by [8] for navigation in low orbits that will be used in NASA's upcoming Magnetospheric Multiscale Mission. Another use is that of the intended application of this research: control of unmanned aerial vehicles for the Defence Science \& Technology Organisation (DSTO) of Australia. Flight trials by the DSTO found that the loss or corruption of GPS signals is a reality. Similar findings were presented in the Volpe report [9]. The Volpe report was prepared by the US Department of Transportation and raised issues regarding GPS vulnerabilities in the US transportation infrastructure. GPS disruptions can occur due to a range of signal interferences, whether intentional such as jamming or spoofing, or unintentional from everyday use of electronic devices such as mobile phones, TV, and more. Subsequently, other methods for controlling formations are required as alternatives to GPS based control.

\section{A. Contribution}

Formation control with bearing-only measurements (and/or desired inter-agent constraints) has been studied in [10]-[18]. Formation control with heterogenous sensing (and/or constraints) has also been considered [19], [20]. Often, with bearing-only sensing (and constraints), the scale of the desired formation is not controllable; e.g. see [11].

In this paper we develop a control system for triangular formations where the motion of each agent is governed by an identical control law and the agents can measure the bearing to other agents and to two landmarks with known positions. Unsurprisingly, with measurement of enough angles, the agents can assemble a picture of the five points, three agents and two landmarks, which is defined up to scaling. Then, any agent can calculate the distance separating itself from another agent by relating this unknown distance to the known distance separating the landmarks (through the measured bearings). Once the inter-agent distances are known then we can define a control law at each agent that forces that agent to achieve and then maintain desired inter-agent distance values. We also extend this distributed control law such that 
each agent maintains the interior angles subtended at itself by the other agents at some desired value (consistent with the desired distances). The end result is an exponentially stable formation control law with a global region of convergence.

As we are concerned with the situation where GPS is unavailable we address the formation shape problem using passive sensors with the requirement that the sensors are capable of measure bearings to objects. Vision-based sensors are a possible platform for implementation. The use of vision-based sensors is studied in [21], and also in [22] where the ground-based agents use catadioptric cameras (i.e. with a wide field of view).

\section{FORMAL PROBLEM STATEMENT}

The problem can be summarised by the following question: how can three bearing-measuring agents control the shape of their triangular formation using two landmarks? In this section we expand and formalise this question.

Consider an undirected graph, $\mathcal{G}$ with the vertex set $\mathcal{V}=\{\mathcal{R}, \mathcal{L}\}$, where $\mathcal{R}=\left\{R_{1}, R_{2}, R_{3}\right\}$, representing the $n=3$ agents; and $\mathcal{L}=\left\{L_{1}, L_{2}\right\}$, representing the $m=2$ landmarks. We suppose the vertices correspond to points in $\mathbb{R}^{2}$. In this paper the agents will be referred to using the subscripts $i, j, k$, and the landmarks with subscripts $s$ and $t$. Note that the agents are treated as point agents.

The agents are equipped with passive sensors capable of measuring the bearing to other agents and landmarks (in a local coordinate frame). Consequently, these measurements are only meaningful in a local sense and are not aligned in any way with those of other agents.

We initially define a desired triangular formation by three lengths. These are the desired inter-agent distances, and are denoted $r_{i, j}^{*}$. The current (or instantaneous) interagent distances are denoted by $r_{i, j}$. We make the following assumptions concerning the current inter-agent positions.

Assumption 1 (Non-collinear or collocated formation). The initial agent positions are not collocated or collinear (i.e. $\left.r_{i, j}<\left(r_{i, k}+r_{j, k}\right), r_{i, j}=\max \left\{r_{i, j}, r_{i, k}, r_{j, k}\right\}\right)$.

Assumption 2 (Non-collinearity with landmarks). No agent lies on the line that intersects both landmarks. Therefore no agents are collinear with the landmarks.

Assumption 1 will be relaxed later in the paper. The goal of this paper is therefore to derive a control law that ensures the convergence of $r_{i, j} \rightarrow r_{i, j}^{*}$ as time, $t \rightarrow \infty$.

An example scenario is shown in Figure 1. It can be noted that all of the edges incident on a given agent connect objects to which the agent is capable of measuring a local bearing.

\section{INTER-AGENT DISTANCE CALCULATION}

Agent $R_{i}$ can measure the bearing $\phi_{i, j}^{R} \in[-\pi, \pi)$ in its own coordinate frame to the neighbouring agent, $R_{j}$, taken from the local $x_{i}$ direction of agent $R_{i}$. Agent $R_{i}$ can also measure the local bearing $\phi_{i, s}^{L} \in[-\pi, \pi)$ to the landmark, $L_{s}$, taken from the local $x_{i}$ direction of agent $R_{i}$. A positive value indicates a bearing taken in an anticlockwise direction and a negative value indicates a clockwise direction.

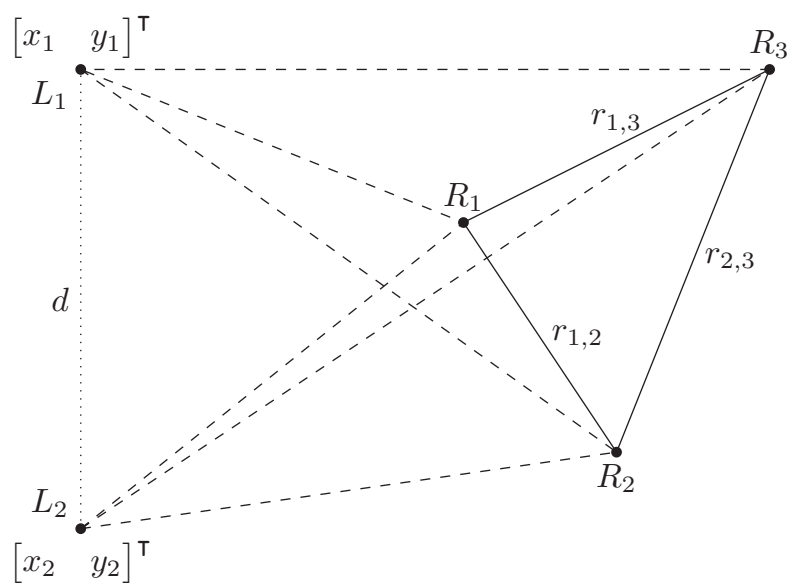

Fig. 1. An example $\mathcal{G}$ showing the edges that connect each of the vertices in $\mathcal{V}$. Note that as the locations of the landmarks are known the distance between them, $d$, can be calculated. This is discussed in Section III.

Let $\vartheta_{i}$ denote the difference between any two bearings, $\phi_{a}$ and $\phi_{b}$, taken by agent $R_{i}$ :

$$
\vartheta_{i}\left(\phi_{a}, \phi_{b}\right)=\left|\phi_{a}-\phi_{b}\right| \in[0,2 \pi) .
$$

Note that this is an angle not a bearing. To use these angles we must ensure that (1) does not settle on the reflex angle. We therefore denote the angle (as seen by agent $R_{i}$ ) between any two given objects as,

$$
\theta_{i}\left(\phi_{a}, \phi_{b}\right)= \begin{cases}\vartheta_{i}\left(\phi_{a}, \phi_{b}\right) & \text { if } \vartheta_{i}\left(\phi_{a}, \phi_{b}\right) \leq \pi \\ 2 \pi-\vartheta_{i}\left(\phi_{a}, \phi_{b}\right) & \text { otherwise }\end{cases}
$$

From the two types of local bearing measurements - to landmarks, $\phi_{i, s}^{L}$, and to other agents, $\phi_{i, j}^{R}$ - three types of angles can be calculated.

$\lambda_{i} \quad$ The angle subtended at agent $R_{i}$ by the two landmarks, $L_{1}$ and $L_{2}: \lambda_{i}=\theta\left(\phi_{i, 1}^{L}, \phi_{i, 2}^{L}\right)$.

$\alpha_{i} \quad$ The angle subtended at agent $R_{i}$ by the two neighbouring agents, $R_{j}$ and $R_{k}: \alpha_{i}=\theta\left(\phi_{i, j}^{R}, \phi_{i, k}^{R}\right)$.

$\eta_{s, j}^{i} \quad$ The angle subtended at agent $R_{i}$ by landmark, $L_{s}$, and agent, $R_{j}: \eta_{s, j}^{i}=\theta\left(\phi_{i, s}^{L}, \phi_{i, j}^{R}\right)$.

The agents can make distance calculations by relating the inter-agent distances to $d$, the distance separating the landmarks. Letting $\boldsymbol{p}_{L_{s}}=\left[\begin{array}{ll}x_{s} & y_{s}\end{array}\right]^{\top} \in \mathbb{R}^{2}$,

$$
d=\left\|\boldsymbol{p}_{L_{1}}-\boldsymbol{p}_{L_{2}}\right\| \text {. }
$$

The length between objects $X$ and $Y$ is denoted by $\overline{X Y}$ where an object is either a landmark or an agent. For example, $d=\overline{L_{1} L_{2}}$ and $r_{i, j}=\overline{R_{i} R_{j}}$.

The law of cosines can be used to describe the triangle that is formed by the edges connecting the two landmarks and agent $R_{i}$ making the measurement:

$$
d^{2}={\overline{L_{1} R_{i}}}^{2}+{\overline{L_{2} R_{i}}}^{2}-2 \overline{L_{1} R_{i}} \overline{L_{2} R_{i}} \cos \left(\lambda_{i}\right)
$$

The unknown distance $\overline{L_{1} R_{i}}$ is related to $r_{i, j}$ through the law of sines as follows:

$$
\overline{L_{1} R_{i}}=\frac{r_{i, j} \sin \left(\eta_{1, i}^{j}\right)}{\sin \left(\gamma_{i, j}^{1}\right)},
$$


where $\gamma_{i, j}^{s}$ is the third interior angle of $\triangle R_{i} R_{j} L_{s}$. Hence,

$$
\gamma_{i, j}^{s}=\pi-\eta_{s, i}^{j}-\eta_{s, i}^{j} .
$$

Likewise, the other unknown distance $\overline{L_{2} R_{i}}$ is related to $r_{i, j}$ also through the law of sines as follows:

$$
\overline{L_{2} R_{i}}=\frac{r_{i, j} \sin \left(\eta_{2, i}^{j}\right)}{\sin \left(\gamma_{i, j}^{2}\right)} .
$$

By substituting (5) and (7), it follows that (4) can be rewritten with only one unknown:

$$
\begin{aligned}
d^{2}= & \left(\frac{r_{i, j} \sin \left(\eta_{1, i}^{j}\right)}{\sin \left(\gamma_{i, j}^{1}\right)}\right)^{2}+\left(\frac{r_{i, j} \sin \left(\eta_{2, i}^{j}\right)}{\sin \left(\gamma_{i, j}^{2}\right)}\right)^{2} \\
& -2\left(\frac{r_{i, j} \sin \left(\eta_{1, i}^{j}\right)}{\sin \left(\gamma_{i, j}^{1}\right)}\right)\left(\frac{r_{i, j} \sin \left(\eta_{2, i}^{j}\right)}{\sin \left(\gamma_{i, j}^{2}\right)}\right) \cos \left(\lambda_{i}\right)
\end{aligned}
$$

A factor of $r_{i, j}^{2}$ can be extracted from each term on the right hand side. This can then be rearranged for $r_{i, j}^{2}$, and by taking the square root we obtain the relationship

$$
r_{i, j}=\sqrt{\frac{d^{2}}{c_{i, j}}},
$$

where

$$
\begin{aligned}
c_{i, j}=\left(\frac{\sin \left(\eta_{s, i}^{j}\right)}{\sin \left(\gamma_{i, j}^{s}\right)}\right)^{2} & +\left(\frac{\sin \left(\eta_{t, i}^{j}\right)}{\sin \left(\gamma_{i, j}^{t}\right)}\right)^{2} \\
& -2\left(\frac{\sin \left(\eta_{s, i}^{j}\right) \sin \left(\eta_{t, i}^{j}\right)}{\sin \left(\gamma_{i, j}^{s}\right) \sin \left(\gamma_{i, j}^{t}\right)}\right)^{2} \cos \left(\lambda_{i}\right) .
\end{aligned}
$$

If Assumptions 1 and 2 hold it is possible for agent $R_{i}$ to calculate the inter-agent distance $r_{i, j}$ using (9) and (10). This method requires the agents to share information with neighbours as $R_{i}$ is incapable of measuring $\eta_{1, i}^{j}$ and $\eta_{2, i}^{j}$. Without such communication, it seems impossible for agent $R_{i}$ to otherwise obtain $r_{i, j}$. The locality of some of the angles required by this method is shown in Figure 2.

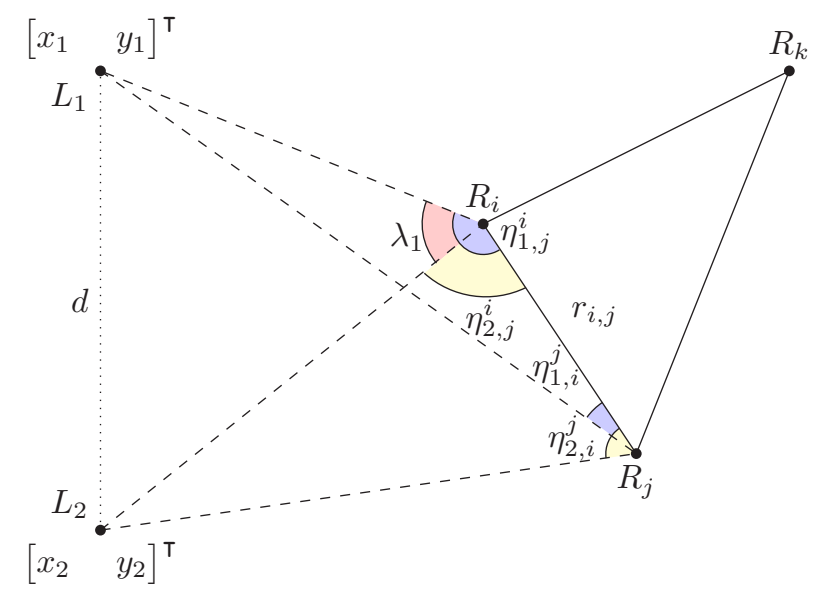

Fig. 2. The angles required by agent $R_{i}$ to make the $r_{i, j}$ inter-agent distance calculation using (9) and (10).

\section{Distance-BASED CONTROL}

Firstly, note that the desired inter-agent distances $r_{i, j}^{*}$ must satisfy the triangular inequality (as in Assumption 1) and we state this inequality explicitly as

$$
r_{i, j}^{*}<\left(r_{i, k}^{*}+r_{j, k}^{*}\right), \quad r_{i, j}^{*}=\max \left\{r_{i, j}^{*}, r_{i, k}^{*}, r_{j, k}^{*}\right\} .
$$

This ensures the existence of a triangle defined by these desired values and bounds the desired formation shape away from any collinear triangle.

\section{A. Proposed Control Law}

The dynamical model of agent $R_{i}$ (in its own coordinate basis) is given by

$$
\dot{p}_{i}=\overline{v_{i}}\left[\begin{array}{l}
\cos \left(\overline{\beta_{i}}\right) \\
\sin \left(\overline{\beta_{i}}\right)
\end{array}\right]
$$

where $\boldsymbol{p}_{i}=\left[\begin{array}{ll}x_{i} & y_{i}\end{array}\right]^{\top} \in \mathbb{R}^{2}$, is the agent's position, and $\overline{v_{i}}$ and $\overline{\beta_{i}}$ are control inputs to be determined. The control inputs for agent $R_{i}$ are defined as,

$$
\overline{\beta_{i}}=\operatorname{atan} 2\left(a_{i}, b_{i}\right),
$$

and,

$$
\overline{v_{i}}=\sqrt{a_{i}^{2}+b_{i}^{2}}
$$

where,

$$
a_{i}=v_{i, j} \sin \left(\phi_{i, j}\right)+v_{i, k} \sin \left(\phi_{i, k}\right)
$$

capturing the vector components in the agent's local $y_{i}$ direction and

$$
b_{i}=v_{i, j} \cos \left(\phi_{i, j}\right)+v_{i, k} \cos \left(\phi_{i, k}\right),
$$

capturing the vector components in the agent's local $x_{i}$ direction, with $v_{i, j}$ defined below ${ }^{3}$.

The movement is proportional to the error in the distance separating the neighbouring agents. Hence the definition of,

$$
v_{i, j}=\left(r_{i, j}^{2}-r_{i, j}^{*}\right) k_{r}
$$

where $k_{r}>0$ is a constant controlling the rate of convergence. Note that the distance measurements are squared to ensure a continuous first derivative.

An agent's movement is effectively determined by a superposition of two actions. The first action is the movement directly towards one neighbouring agent if the desired interagent distance is smaller than the current distance (else directly away from that neighbour). The second is a similar movement either directly towards or away from the other neighbouring agent. Hence, the dynamics of the agent are practically equivalent to the following mathematical model

$$
\dot{p}_{i}=v_{i, j}\left[\begin{array}{c}
\cos \left(\phi_{i, j}\right) \\
\sin \left(\phi_{i, j}\right)
\end{array}\right]+v_{i, k}\left[\begin{array}{c}
\cos \left(\phi_{i, k}\right) \\
\sin \left(\phi_{i, k}\right)
\end{array}\right] .
$$

\footnotetext{
${ }^{3}$ The function $\operatorname{atan} 2(y, x)$ that is used in (13) computes $\arctan \left(\frac{y}{x}\right)-$ equivalently denoted $\tan ^{-1}\left(\frac{y}{x}\right)$ - while accounting for the quadrant within which this angle lies. Thus atan $2(y, x)$ lies in the interval $(-\pi, \pi]$ whereas $\arctan \left(\frac{y}{x}\right)$ lies in $\left(\frac{-\pi}{2}, \frac{\pi}{2}\right]$. By definition $\operatorname{atan} 2(0,0)=0$. The quadrant is determined by the signs of both arguments $x$ and $y$. This function is commonly provided by programming languages including MATLAB.
} 
While (18) illustrates the motion of an agent in a more intuitive manner we must remember that each agent has only a single-integrator dynamic model with only a single speed and heading input. Thus, when defining the control inputs we must do so with (12) in mind as initially indicated above.

\section{B. Dynamics and stability}

This section analyses the dynamic system using the state variables $r_{1,2}{ }^{2}, r_{1,3}{ }^{2}$, and $r_{2,3}{ }^{2}$. The state variables are represented in vector form by

$$
x=\left[\begin{array}{lll}
r_{1,2}^{2} & r_{1,3}^{2} & r_{2,3}^{2}
\end{array}\right]^{\top} .
$$

In addition we define a similar vector of desired values,

$$
x^{*}=\left[\begin{array}{lll}
r_{1,2}^{*}{ }^{2} & r_{1,3}^{*}{ }^{2} & r_{2,3}^{*}{ }^{2}
\end{array}\right]^{\top} .
$$

From these vectors the system dynamics are governed by

$$
\dot{x}=\boldsymbol{A}\left(x-x^{*}\right),
$$

where the $3 \times 3$ square matrix $\boldsymbol{A}$ is derived below.

There are four movements that govern the dynamics of each state variable. These arise from agent $R_{i}$ 's movement to control $r_{i . j}$ and $r_{i . k}$ to the desired values, and from $R_{j}$ 's movement to control $r_{i . j}$ and $r_{j . k}$ to the desired values. Note that the movements of agent $R_{k}$ do not directly affect these dynamics.

The two components causing a change in $r_{i . j}$ from the movements of the two neighbouring agents moving directly towards or away from one another are given by,

$$
\dot{r}_{i, j}=\left(r_{i, j}^{2}-r_{i, j}^{2}\right) k_{r} .
$$

The other two components are given by agent $R_{i}$ 's and agent $R_{j}$ 's movements directly towards or away from agent $R_{k}$. These components are given respectively below,

$$
\begin{gathered}
\dot{r}_{i, j}=\left(r_{i, k}{ }^{2}-r_{i, k}^{*}{ }^{2}\right) k_{r} \cos \left(\alpha_{i}\right), \quad \text { and }, \\
\dot{r}_{i, j}=\left(r_{j, k}{ }^{2}-{r_{j, k}^{*}}^{2}\right) k_{r} \cos \left(\alpha_{j}\right) .
\end{gathered}
$$

As the state variables are squared values, we must relate the components derived above to the state variable. As the time derivative of $r_{i, j}^{2}$ is $r_{i, j}^{2}=2 r_{i, j} r_{i, j}$ we can write the above components into the form of (21) and define the matrix $A$ :

$$
\boldsymbol{A}=2 k_{r}\left[\begin{array}{clrlrl}
2 & r_{i, j} & r_{i, j} & \cos \left(\alpha_{1}\right) & r_{i, j} & \cos \left(\alpha_{2}\right) \\
r_{i, k} & \cos \left(\alpha_{1}\right) & 2 & r_{i, k} & r_{i, k} & \cos \left(\alpha_{3}\right) \\
r_{j, k} & \cos \left(\alpha_{2}\right) & r_{j, k} & \cos \left(\alpha_{3}\right) & 2 & r_{j, k}
\end{array}\right]
$$

Now define the control error $e=x-x^{*}$ such that the following error system is easily derived

$$
\dot{e}=\boldsymbol{A} e
$$

Then the following result follows.

Theorem 1. Suppose Assumptions 1 and 2 hold and that $\alpha_{i} \in(0, \pi)$ and $r_{i, j}>0, \forall i, j$ for some $t_{0}$. Then solutions to $\dot{e}=\boldsymbol{A} e$ exist for all $t \in\left[t_{0}, \infty\right)$. Further, the equilibrium $e=0$ of the error system (26) is exponentially stable.
Proof: Consider the Lyapunov function

$$
v=e^{\top} e
$$

Then $\dot{v}=e^{\top} \boldsymbol{A} e$. Pick a row of $\boldsymbol{A}$ and we conclude that $\boldsymbol{A}$ is negative-semidefinite via diagonal dominance since $\alpha_{i} \in$ $[0, \pi]$. Thus, solutions exist for all $t \in\left[t_{0}, \infty\right)$. Further, $\boldsymbol{A}$ is strictly negative-definite whenever $\alpha_{i} \in(0, \pi)$.

Now note that the dynamics (18) of each agent are qualitatively equivalent to the dynamics of those agents driven by so-called rigidity-based formation control laws; see [23][25]. In [26] it is proven that a formation obeying such dynamics and initialised at $t_{0}$ with $\alpha_{i} \in(0, \pi)$, i.e. with $\boldsymbol{A}<0$ will ensure that $\boldsymbol{A}$ retains strict negative-definiteness $\boldsymbol{A}<0$ for all $t \in\left[t_{0}, \infty\right)$. Therefore, exponential stability follows; see also [27].

We thus have an exponentially stable control law for formation shape and scale control with agents capable of bearing-only sensing. The control law thus far permits any initial formation shape besides those in which the agents are collinear or collocated and it permits any desired formation shape besides a collinear formation shape. We will relax this initialisation restriction later.

\section{Simulation}

We simulated this control law using MATLAB. The initial and final triangular formations as well as the landmark locations were randomly generated. One such simulation is illustrated in Figures 3 and 4.

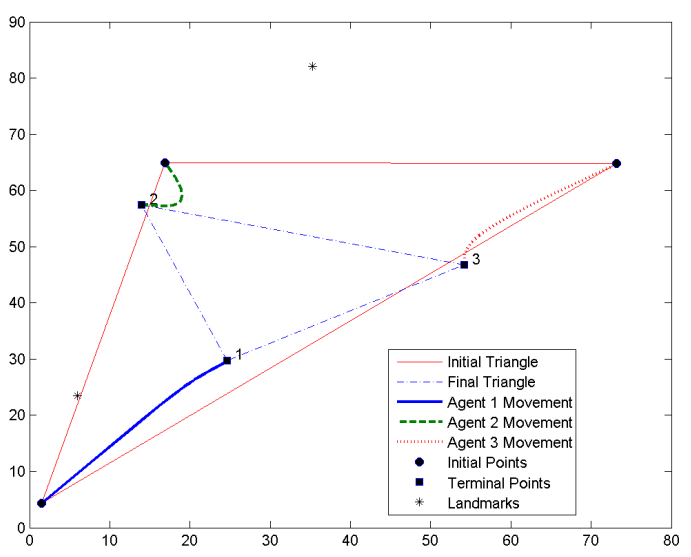

Fig. 3. The initial and final triangular formation with the proportional gain $k_{r}$ set to 0.0001 . The lines connecting the triangles show the path taken by each of the agents as they converge upon the desired shape.

\section{EXTENSION TO INCLUDE COLLINEAR FORMATIONS}

The control law derived above can be used for triangular formations where the initial agent positions satisfy Assumptions 1 and 2 and the desired formation satisfies

$$
r_{i, j}^{*}<\left(r_{i, k}^{*}+r_{j, k}^{*}\right), \quad r_{i, j}^{*}=\max \left\{r_{i, j}^{*}, r_{i, k}^{*}, r_{j, k}^{*}\right\} .
$$

However, it is possible to relax the initial non-collinear requirement of Assumption 1. In this section we derive 


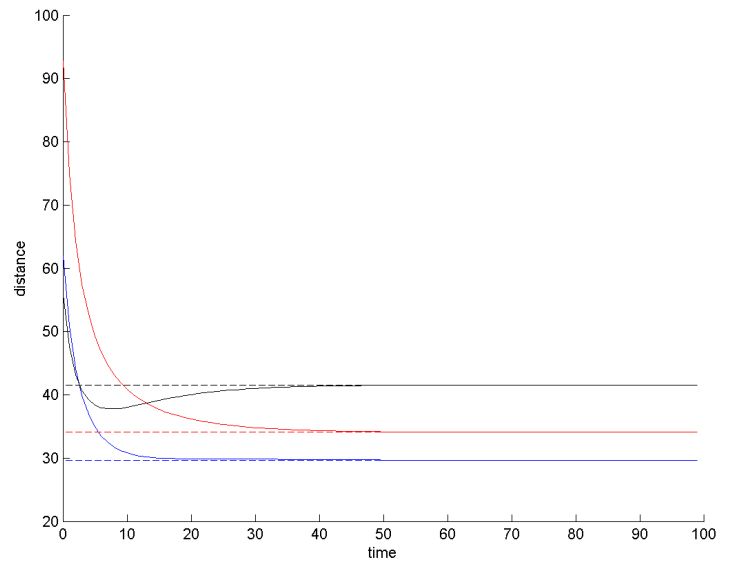

Fig. 4. Convergence of the inter-agent distances to the desired values.

another control law that can push the agents away from a collinear formation. Mathematically we have relaxed the triangular inequality for the initial formation to

$$
r_{i, j} \leq\left(r_{i, k}+r_{j, k}\right), \quad r_{i, j}=\max \left\{r_{i, j}, r_{i, k}, r_{j, k}\right\} .
$$

Note that the final formation as specified by the set of desired inter-agent distances must still satisfy (28).

Using (2) we can calculate the interior angle of the formation, $\alpha_{i}$, at agent $R_{i}$. This is the angle subtended at agent $R_{i}$ by the two neighbouring agents, $R_{j}$ and $R_{k}$ : $\alpha_{i}=\theta\left(\phi_{i, j}^{R}, \phi_{i, k}^{R}\right)$. We use this to derive a new control law that directly acts on the errors between the actual and desired interior angles by moving the agents to reduce the errors.

For this new control law we define three desired interior angles, $\alpha_{i}^{*}, \alpha_{j}^{*}$, and $\alpha_{k}^{*}$, such that,

$$
\sum_{i=1}^{n} \alpha_{i}^{*}=\pi, \quad \text { where } \alpha_{i}^{*} \in[0, \pi] .
$$

We require that $\alpha_{i}^{*} \neq 0$ for any $i$. The desired angles capture the shape but not scale of the desired triangular formation. They can be calculated from the desired inter-agent distances using the law of cosines,

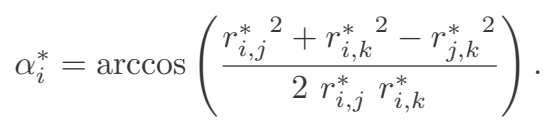

and obviously such an equation must be satisfied; i.e. the desired interior angles must be consistent with the desired inter-agent distances.

\section{A. An Extension to the Proposed Control Law}

The extended control law governing the motion of agent $R_{i}$ is given below. It takes the form of a switching controller that switches between two laws. One of the control laws is the law defined in Section IV-A. However, as this control law will not work when the agents are collinear we thus derive a second law to address this situation. The second law works on the set of interior angles subtended at each agent by its neighbours. The extension is based on [10], [11].
In order to use this extension a small angle $\epsilon$ must be defined where $0<\epsilon<\pi$. Ideally $\epsilon$ should be closer to 0 . This is used by the agents to decide which law is used at each moment.

The extended control law is given by

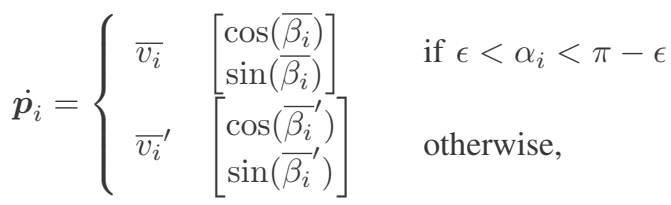

with the control inputs to the first case derived in Section IV$\mathrm{A}$ and the control inputs to the second case derived below.

The direction of the vector $\dot{p}_{i}$ in the second case is

$$
{\overline{\beta_{i}}}^{\prime}=\left\{\begin{array}{cl}
\frac{\alpha_{i}}{2}+\min \left(\phi_{i, j}, \phi_{i, k}\right) & \text { if } \vartheta_{i}\left(\phi_{i, j}, \phi_{i, k}\right) \leq \pi \\
\frac{\alpha_{i}}{2}+\max \left(\phi_{i, j}, \phi_{i, k}\right) & \text { if } \vartheta_{i}\left(\phi_{i, j}, \phi_{i, k}\right)>\pi,
\end{array}\right.
$$

where $\vartheta_{i}$ is given by (1). The speed ${\overline{v_{i}}}^{\prime}$ is proportional to the error in the angle $\alpha_{i}$ from its desired value,

$$
{\overline{v_{i}}}^{\prime}=-\left(\alpha_{i}-\alpha_{i}^{*}\right) k_{\alpha}, \quad k_{\alpha}>0 .
$$

If the current formation is collinear then the effect of this control law is to drive the agents away from collinearity and then let the original distance-based control law take over. Once this happens we have it from Theorem 1 that the control law is exponentially stable. Thus, for all noncollinear desired triangles there exists an appropriate $\epsilon$ such that we have an effective global stability result.

\section{B. Simulations}

We now provide simulations using this new control law where the initial agent formation is collinear. The results of the simulation are illustrated in Figure 5. We note that by controlling the interior angles of the formation in addition to controlling the inter-agent distances the agents are able to move from a collinear formation to a triangular formation.

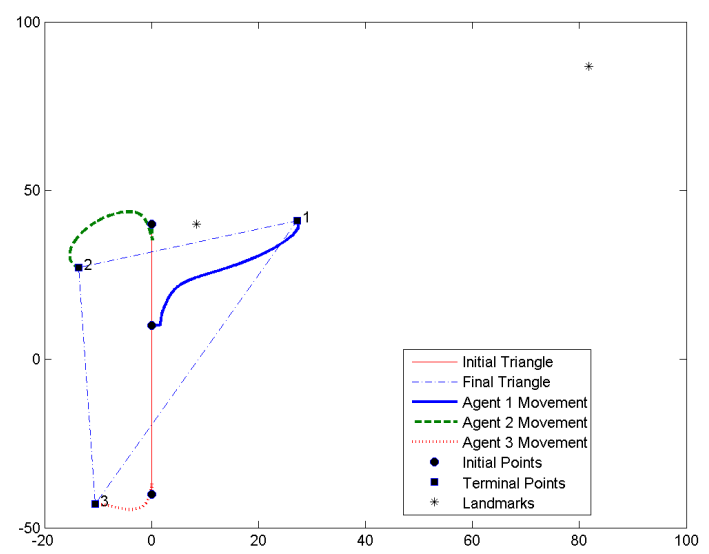

Fig. 5. The initial and final triangular formations with the $k_{\alpha}=1, k_{r}=$ 0.001 and $\epsilon=5^{\circ}$. Agents are driven from their collinear starting point. 


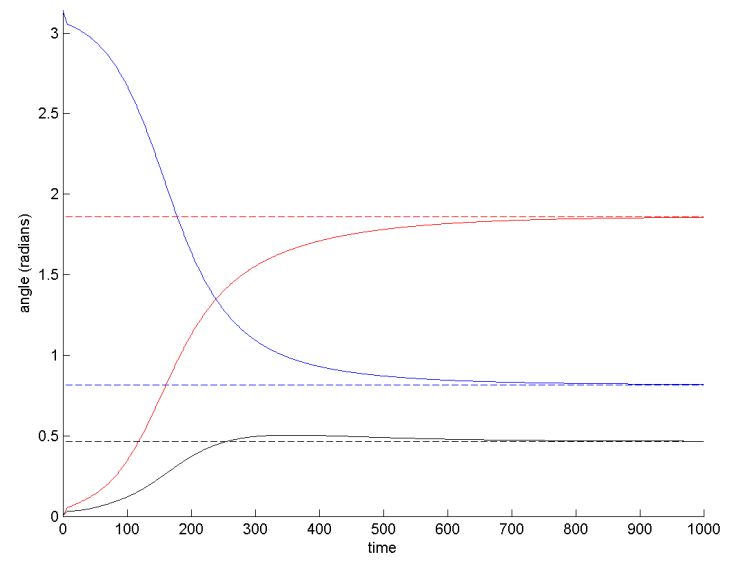

Fig. 6. The inter-agent angles tend to their desired values as the agents converge to the desired formation.

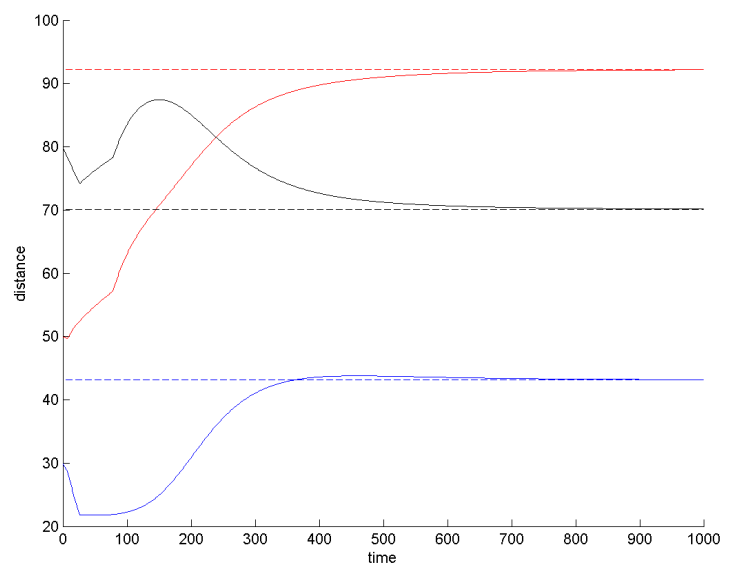

Fig. 7. The inter-agent distances tend to their desired values as the agents converge to the desired formation.

\section{CONCLUSION}

In this work we defined a law for controlling the shape and scale of triangular formations of agents with (local) bearingonly sensing and landmarks with known positions.

\section{REFERENCES}

[1] K. Oh, M. Park, and H. Ahn, "A survey of multi-agent formation control," Automatica, vol. 53, no. 3, pp. 424-440, March 2015.

[2] A. Bishop, B. Fidan, B. Anderson, K. Dogancay, and P. Pathirana, "Optimality analysis of sensor-target localization geometries," Automatica, vol. 46, no. 3, pp. 479-492, March 2010.

[3] A. Bishop and P. Jensfelt, "An optimality analysis of sensor-target geometries for signal strength based localization," in Proc. of the 5th International Conference on Intelligent Sensors, Sensor Networks and Information Processing (ISSNIP'09), Melbourne, December 2009.

[4] C. W. Reynolds, "Flocks, herds, and schools: A distributed behavioral model," in 14th Annual Conference on Computer Graphics and Interative Techniques, July 1987, pp. 25-34.

[5] T. Vicsek, A. Czirók, E. Ben-Jacob, I. Cohen, and O. Shochet, "Novel type of phase transition in a system of self-driven particles," Physical Review Letters, vol. 75, no. 6, pp. 1226-1229, August 1995.
[6] A. Jadbabaie, J. Lin, and A. S. Morse, "Coordination of groups of mobile autonomous agents using nearest neighbor rules," IEEE Trans. on Automatic Control, vol. 48, no. 6, pp. 988-1001, June 2003.

[7] J. Curcio, J. Leonard, J. Vaganay, A. Patrikalakis, A. Bahr, D. Battle, H. Schmidt, and M. Grund, "Distributed optimization for cooperative agents: Application to formation flight," in Proceedings of the MTS/IEEE Oceans, September 2005, pp. 730-735.

[8] L. M. B. Winternitz, W. A. Bamford, and G. W. Heckler, "A GPS receiver for high-altitude satelliite navigation," IEEE Journal of Selected Topics in Signal Processing, vol. 3, no. 4, pp. 988-1001, August 2009.

[9] J. V. Carroll, "Vulnerability assessment of the US transportantion infrastructure that relies on the global positioning system," Journal of Navigation, vol. 56, pp. 185-193, 2003.

[10] A. Bishop and M. Basiri, "Bearing-only triangular formation control on the plane and the sphere," in Proc. of the 18th Mediterranean Conf. on Control and Automation, Marrakech, Morocco, June 2010.

[11] M. Basiri, A. Bishop, and P. Jensfelt, "Distributed control of triangular formations with angle-only constraints," Systems and Control Letters, vol. 59, no. 2, pp. 147-154, February 2010.

[12] A. Bishop, "A very relaxed control law for bearing-only triangular formation control," in Proc. of the 2011 IFAC World Congress, Milan, Italy, August 2011.

[13] — , "Distributed bearing-only quadrilateral formation control," in Proc. of the 18th IFAC World Congress, Milan, Italy, August 2011.

[14] A. Bishop, I. Shames, and B. Anderson, "Stabilization of rigid formations with direction-only constraints," in Proc. of the 50th IEEE Conference on Decision and Control and European Control Conference (CDC-ECC 2011), Orlando, Florida, December 2011.

[15] A. Bishop, "Distributed bearing-only formation control with four agents and a weak control law," in Proc. of the 9th IEEE International Conference on Control and Automation, Santiago, Chile, December 2011, pp. 30-35.

[16] A. Franchi and P. Giordano, "Decentralized control of parallel rigid formations with direction constraints and bearing measurements," in Proc. of the 51st IEEE Annual Conference on Decision and Control, December 2012, pp. 5310-5317.

[17] S. Zhao, F. Lin, K. Peng, B. Chen, and T. Lee, "Distributed control of angle-constrained cyclic formations using bearing-only measurements," Systems and Control Letters, vol. 63, pp. 12-24, 2014.

[18] S. Zhao and D. Zelazo, "Bearing rigidity and almost global bearingonly formation stabilization," arXiv preprint arXiv:1408.6552, 2014.

[19] A. Bishop, T. Summers, and B. Anderson, "Control of triangle formations with a mix of angle and distance constraints," in Proc. of the 2012 Multi-conference on Systems and Control, Dubrovnik, Croatia, October 2012

[20] _ "Stabilization of stiff formations with a mix of direction and distance constraints," in Proc. of the 2013 Multi-conference on Systems and Control, Hyderabad, India, August 2013, pp. 1194-1199.

[21] N. Moshtagh, N. Michael, A. Jadbabaie, and K. Daniilidis, "Visionbased, distributed control laws for motion coordination of nonholonomic robots," IEEE Transactions on Robotics, vol. 25, no. 4, pp. 851-860, August 2009.

[22] A. K. Das, R. Fierro, V. Kumar, J. P. Ostrowski, J. Spletzer, and C. J. Taylor, "A vision-based formation control framework," IEEE Transactions on Robotics and Automation, vol. 18, no. 5, pp. 813825, October 2002

[23] R. Olfati-Saber and R. Murray, "Graph rigidity and distributed formation stabilization of multi-vehicle systems," in Proc. of the 41st IEEE Conference on Decision and Control, Las Vegas, Nevada, USA, 2002, pp. 2965-2971.

[24] L. Krick, M. Broucke, and B. Francis, "Stabilisation of infinitesimally rigid formations of multi-robot networks," International Journal of Control, vol. 82, no. 3, pp. 423-439, 2009.

[25] A. Bishop, M. Deghat, B. Anderson, and Y. Hong, "Distributed formation control with relaxed motion requirements," International Journal of Robust and Nonlinear Control, vol. 25, no. 17, pp. 32103230, November 2015.

[26] Z. Sun, U. Helmke, and B. Anderson, "Rigid formation shape control in general dimensions: an invariance principle and open problems," in Proc. of the 54th IEEE Conference on Decision and Control, Tokyo, Japan, December 2015.

[27] Z. Sun, S. Mou, B. Anderson, and A. Morse, "Non-Robustness of Gradient Control for 3-D Undirected Formations with Distance Mismatch," in Proc. of the 2013 Australian Control Conference, Perth, Australia, November 2013. 